\title{
SMALL-X QCD EFFECTS IN PARTICLE COLLISIONS AT HIGH ENERGIES
}

\author{
TANCREDI CARLI \\ DESY, Notkestr.85, Hamburg Germany, E-mail: carli@mail.desy.de \\ Invited talk at the XX. Int. Sym. on Lepton and Photon Interactions at High Energies, \\ Rome, Italy, July 2001
}

\begin{abstract}
Recent theoretical developments to calculate cross sections of hadronic objects in the high energy limit are summarised and experimental attempts to establish the need for new QCD effects connected with a resummation of small hadron momentum fractions $x$ are reviewed. The relation between small- $x$ parton dynamics and the phenomenon of diffraction is briefly out-lined. In addition, a search for a novel, non-perturbative QCD effect, the production of QCD instanton induced events, is presented.
\end{abstract}

\section{Mini-Introduction to Recent BFKL Progress}

When two hadronic objects collide at high energies, $h_{1} h_{2} \rightarrow h_{3} h_{4} X$, often more than one energy scale is involved. Of particular interest are collisions where the squared centre of mass $(\mathrm{cm})$ energy $s=\left(h_{1}+h_{2}\right)^{2}$ is much larger than the squared transverse momentum transfer $t=\left(h_{1}-h_{3}\right)^{2}$, i.e. $s \gg t \gg$ $\Lambda_{Q C D}$. In perturbative QCD (pQCD) calculations of such processes large logarithms of the ratio of these scales, $\left(\alpha_{s} \ln (s / t)\right)^{n}$, arise at each order $n$ in the strong coupling constant $\alpha_{s}$. It is usually assumed that they must be resummed to obtain a reliable cross section prediction.

About 25 years ago, Balitsky, Fadin, Kuraev and Lipatov (BFKL) derived in the leading logarithmic approximation (LLA) an equation describing the resummation of a certain class of exchanges with one chain of multiple gluons in the $t$-channell. It predicts a power-law rise of the scattering cross section at high energies:

$$
\sigma_{B F K L}^{L O} \propto s^{\lambda} \text { with } \lambda=\frac{\alpha_{s}}{\pi} 12 \ln 2
$$

where $\alpha_{s}$ is taken at the value of a hard scale involved in the process. Since for typical values of $\alpha_{s}$, e.g. $\alpha_{s}=0.2, \lambda \approx 0.5$, the predicted rise of the cross section is so strong that the unitarity bound will be violated. This is an indication that at high energies novel effects like parton recombination or multiple perturbative scatterings taming down the rise should be important. Over the

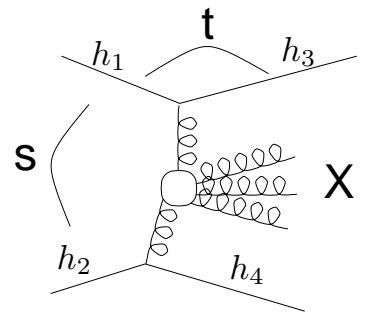

Figure 1. Sketch of a high energy collision of two hadronic objects $h_{1} h_{2} \rightarrow h_{3} h_{4} X$.

past years much experimental effort has been devoted to observe to such phenomena. Although some hints for a rise of the scattering cross section at high energies have been found, the predicted large value of $\lambda$ could not be confirmed.

After a huge theoretical effort by many authors, the calculations of the next-toleading order (NLO) corrections to the BFKL equations were finalised in 1998. The NLO terms are suppressed by a power of $\alpha_{s}$ : $\alpha_{s}\left(\alpha_{s} \ln (s / t)\right)^{n}$. To the consternation of the community, the NLO corrections turned out to be large and lead to much smaller values of $\lambda$ than the leading order (LO) contribution. Fig. 2 shows $\lambda$ calculated in LO and NLO as a function of $\alpha_{s}$. For instance, for a scale where $\alpha_{s} \approx 0.16$ the NLO corrections exactly cancel the LO term. For larger values, $\lambda$ even becomes negative. Naively, this would lead to a cross section that decreases rather than increases as a power of $s$. This is in contradiction to the data. The large NLO corrections put the applicability of the BFKL resummation in question. It seems necessary to understand the physical reason for the large corrections and to include them at all orders. 


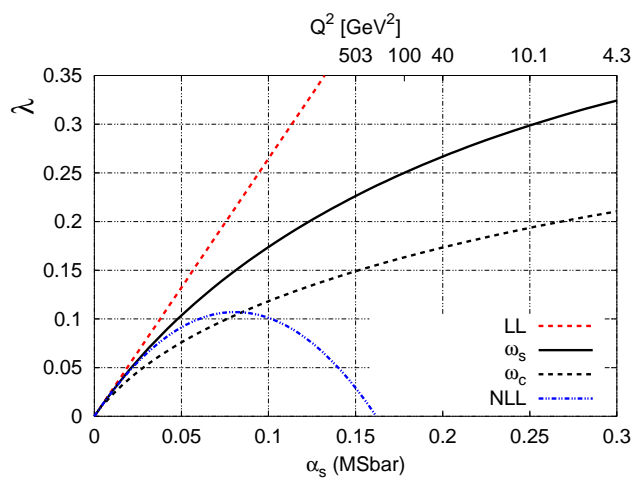

Figure 2. The BFKL exponent $\lambda$ as a function of $\alpha_{s}$ in the LO and NLO approximation and the collinearly enhanced resummation included at NLO.

Several solutions have been proposed to solve this problem 3 A, all of which are related to modelling and to resumming the higher order contributions. One promising method is to analyse the structure of the divergences of the BFKL characteristic function ${ }^{a}$ at all orders by studying the limit where partons are emitted collinearly enhanced contributions have been shown to be able to estimate the NLO corrections and there are good reasons to believe that they also might give a significant contribution of the higher order corrections beyond NLO. The exponents obtained from this collinear resummation are shown in Fig. 2. The label $\omega_{s}$ is related to the power of the cross section expected for $\gamma^{*} \gamma^{*}$ collisions or jet observables in collisions of hadronic objects. The line labelled $\omega_{c}$ is the leading pole in the anomalous dimension of the gluon. The difference between the two exponents does not reflect an additional uncertainty, but is caused by additional corrections for quantities involving an effective cut-off on the lowest accessible momentum necessary to avoid diffusion into the infra-red and non-perturbative region and is closely related to differences in running coupling effects. The resummation

${ }^{a}$ The characteristic function is the Mellin transform of the BFKL kernel. The anomalous dimension is the Mellin transform of the splitting function. See ref 5 for an introduction to more details.

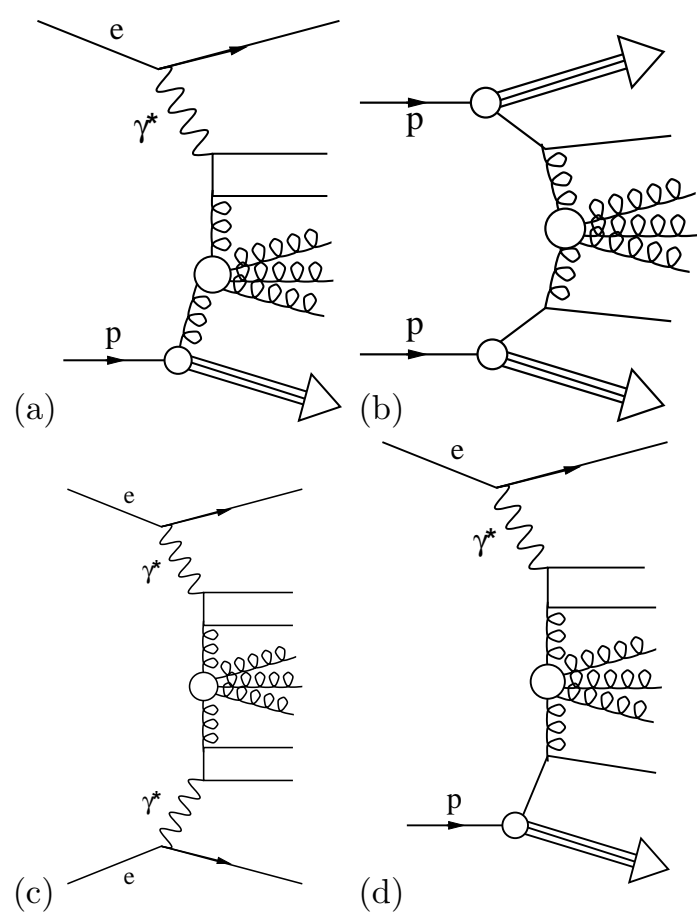

Figure 3. Sketch of small- $x$ signatures in high energy collisions: a) total cross section in DIS, b) dijet production in $p \bar{p}$ collisions, c) total hadronic cross section in $\gamma^{*} \gamma^{*}$ collisions d) forward jet production in DIS.

in the collinear limits leads to values of $\lambda$ which are in qualitative agreement with the experimental observations (see later). However, a quantitative analysis is still lacking.

Although most of the theoretical effort was devoted to understanding the BFKL behaviour at NLO, most of the phenomenological studies still use LO. For instance, only recently part of the virtual photon impact factor, i.e. the coupling of the photon to the gluon chain, has been calculated in $\mathrm{NLO}_{12}$.

\section{Parton Evolution and DIS}

In deep-inelastic scattering (DIS)円, a photon with virtuality $Q^{2} \gg m_{p}^{2}$, where $m_{p}$ is the proton mass, collides with a proton. When the squared $\gamma^{*} p$ cm energy $W^{2}$ is much larger than $Q^{2}$, the momentum fraction of the struck quark, $x \approx Q^{2} / W^{2}$, becomes small.

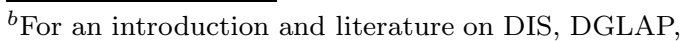
small- $x$ and related topics see:

http://sesam.desy.de/ ح carli/hera.html
} 
In this case, the simple picture of DIS as a process where a virtual photon interacts instantaneously with a point-like parton quark freely moving in the proton has to be modified. Indeed, the probability that a gluon radiates becomes increasingly high at small- $x$ and therefore the quark struck by the photon originates from a cascade initiated by a parton with high longitudinal momentum. A sketch of such an interaction is shown in Fig. 3a). The gluon is the driving force behind the cascade indicated by the circle.

There are three equations describing the parton evolution in different kinematic regions: DGLAP, BFKL and CCFM equations.

\subsection{DGLAP and DLL Evolution}

To correctly calculate the inclusive DIS cross section (for all $x$-values), terms of the form $\left(\alpha_{s} \ln \left(Q^{2} / Q_{0}^{2}\right)\right)^{n}$ have to be resummed, since the smallness of $\alpha_{s}$ is compensated by the large size of $\ln Q^{2}$. This resummation can be achieved by the DGLAP equationst. They are related to the renormalisation group equation and describe the change of the parton density in the proton with varying spatial resolutions $Q^{2}$. Once the parton distribution is specified at a given scale $Q_{0}^{2}$, it can be predicted for any other $Q^{2}$. In an appropriate gauge, the subsequent parton emissions from the proton to the struck quark (connecting the soft proton constituents to the hard subprocess) can be interpreted as a ladder diagram whose rungs and emitted partons are strongly ordered in transverse momentum $k_{T}$. This leads to a suppression of the available phase space for gluon radiation.

For high energy scattering, i.e. at small$x$, the ladder becomes long and consists dominantly of gluons ${ }^{\text {a }}$. The correct description

${ }^{c} k_{T}$ ordering is an assumption necessary to derive the DGLAP equations. For an introduction see ref.

${ }^{d}$ The splitting function $P_{g g}(z)$ (describing the radiation of gluons from gluons) dominates at small longitudinal momentum fractions $z\left(P_{g g}(z) \sim 1 / z\right.$ while $P_{q q}(z) \sim$ constant). $z$ ranges between $x$ and 1. of the small- $x$ region is still a matter of debate and subject to many technical difficulties. The dominant terms are of the form $\left(\alpha_{s} \ln (1 / x) \ln \left(Q^{2} / Q_{0}^{2}\right)\right)^{n}$. Their resummation is referred to as the double-leading logarithmic approximation (DLL). Both longitudinal and transverse momenta are strongly ordered. The DLL leads to a gluon density strongly rising with an effective power $\lambda \approx \sqrt{\left(12 \alpha_{s} / \pi\right) \ln (1 / x) \ln \left(Q^{2} / Q_{0}^{2}\right)}$ towards low- $x$. $\lambda$ depends on $x$ and $Q^{2}$, e.g. it increases towards higher $Q^{2}$.

\subsection{BFKL Evolution}

For $Q^{2} \approx Q_{0}^{2}$, double logarithms no longer dominate and the terms $\left(\alpha_{s} \ln (1 / x)\right)^{n} \approx$ $\left(\alpha_{s} \ln \left(W^{2} / Q^{2}\right)\right)^{n}$ are important and must be resummed using the BFKL equation. In a physical gauge, these terms correspond to an $n$-rung ladder diagram in which gluon emissions are ordered in longitudinal momentum, $z$. The strong $k_{T}$ ordering is replaced by a diffusion pattern as one proceeds along the gluon chain. The BFKL equations describe how a particular high momentum parton in the proton is dressed by a cloud of gluons at small- $x$ localised in a fixed transverse spatial region of the proton given by $Q^{2}$.

A framework to describe the inclusive DIS cross section at small- $x$ is a $k_{T}$ dependent, unintegrated, gluon density $f\left(z, k_{T}\right)$ :

$$
\sigma \propto \int \frac{d z}{z} d^{2} k_{T} \hat{\sigma}\left(x / z, k_{T}\right) f\left(z, k_{T}\right)
$$

where $\hat{\sigma}\left(x / z, k_{T}\right)$ is the partonic cross section. Together with the $k_{T}$ factorisation 10 this forms a basis for a general calculation scheme for small- $x$ processes.

At very small- $x$, it is expected that many gluons coexist and will no longer act as free partons, but interact with each other. This "saturation" regime is characterised by an equilibrium of gluon emission and absorption. In the regime accessible for HERA such effects are expected to be small 3 , but could be rather large when lower $x$-values could be reached. At THERA colliding the $500 \mathrm{GeV}$ 
electrons from TESLA with the $920 \mathrm{GeV}$ proton from HERA such effects will be visible. Also if HERA collided heavy ions instead of protons, an experimental study of saturation effects would probably be possible.

\subsection{CCFM Evolution}

The CCFM11 evolution equation is based on angular ordering and colour coherence. As a result in the appropriate limit it reproduces the DGLAP and the BFKL approximation. The angular ordering of the emitted partons in the initial cascade results from colour coherence and acts as unifying principle 9 . At small values of the parton momentum fractions $z$, a rapdom $k_{T}$ walk is obtained. A first attempt 14 to incorporate the CCFM equations in a Monte Carlo simulation program was not suited for practical purposes. Later a further analysis 15 to make use of the CCFM equations for small$x$ phenomenology showed the importance of the inclusion of soft corrections and revealed many theoretical difficulties, in particular with terms diverging for high rather than low momentum fraction $z$. Recently, significant progress has been made to correctly implement the CCFM evolution equations and to efficiently generate unweighted events using the backward evolution in Monte Carlo simulation program CASCADE 16.17. A slightly differentapproach is followed in the LDC program 18 . Both programs are able to make predictions for any physical observable based on the hadronic final state. Only gluoninitiated processes can be simulated up to now. Some problems still remain, e.g. so far as in the CCFM evolution only the singular terms in the splitting functions (for $z \rightarrow 0$ and $z \rightarrow 1$ ) have been considered.

${ }^{e}$ From the proton $p$ to the photon in the collinear DGLAP approximation $k_{T}$ and therefore the angle increases, while in the BFKL approximation the angle increases because of the decreasing longitudinal momenta $\left(\theta \approx k_{T} /(z p)\right)$. See ref. 13 for an introduction to angular ordering as interpolation between DGLAP and BFKL.

\section{Small- $x$ Signatures in High Energy Collisions}

Several signatures for small- $x$ QCD effects have been recently investigated: jets at large rapidity in $p \bar{p}$ collisions at TEVATRON, the total hadronic cross section in $\gamma^{*} \gamma^{*}$ collisions at LEP and the rise of $F_{2}$ towards small- $x$ and the production of forward jets and particles in $e p$ collisions at HERA.

\subsection{Jet Production at Large Rapidity in $p \bar{p}$ Collisions}

In the high energy limit of $p \bar{p}$ collisions inclusive dijet production (see Fig. 3b) is sensitive to large logarithms of the form:

$$
\sigma \propto \exp \left(\lambda \ln \frac{W^{2}}{E_{T, 1} E_{T, 2}}\right) \propto \exp (\lambda \Delta \eta)
$$

where $Q^{2}=E_{T, 1} E_{T, 2}$ is the momentum transfer to the hard scattering and $W^{2}=$ $x_{1} x_{2} s$ is the squared cm energy of the hard scattering process $\mathrm{f}$. The last proportionality in equation 2 follows after some calculations for $\Delta \eta \gg 1$ from

$$
x_{i}=\frac{2 E_{T, i}}{\sqrt{s}} \exp (\bar{\eta}) \cosh \frac{\Delta \eta}{2}
$$

with $i=1,2$ and $\bar{\eta}=\left(\eta_{1}+\eta_{1}\right) / 2$ and $\Delta \eta=$ $\left|\eta_{1}-\eta_{2}\right|$ for the most backward $\left(\eta_{1}\right)$ and the most forward $\left(\eta_{2}\right)$ jet. Equation 3 is only valid for 2-body kinematics.

The largest logarithms are therefore enhanced for minimal jet $E_{T}$ and maximal $W$. To avoid the strong dependence on the steeply falling parton densities at small- $x$, it has been proposed by Mueller and Navelet 19 to study the dijet cross section at fixed $x_{i}$ at different $\mathrm{cm}$ energies $s$. In an analysis performed by D020, jets have been selected by $E_{T, i}>20 \mathrm{GeV}$ and $\left|\eta_{i}\right|<3$. Moreover, $400<\left(Q^{2}=E_{T, 1} E_{T, 2}\right)<1000 \mathrm{GeV}^{2}$ was

\footnotetext{
$f$ Here $E_{T, i}$ are the transverse jet energies, $\eta_{i}$ the pseudo-rapidities and $x_{i}$ the momentum fractions of the two incoming partons.
} 
demanded. The cross section ratio at fixed $x_{i}$ for two $\mathrm{cm}$ energies has been measured:

$$
R=\frac{\sigma\left(\sqrt{s_{a}}\right)}{\sigma\left(\sqrt{s_{b}}\right)}=\frac{\exp \left(\lambda\left(\Delta \eta_{a}-\Delta \eta_{b}\right)\right)}{\sqrt{\Delta \eta_{a} / \Delta \eta_{b}}}
$$

The result of the cross section ratio at $\sqrt{s_{a}}=$ $1.8 \mathrm{TeV}$ and $\sqrt{s_{b}}=630 \mathrm{GeV}$ is shown as a function of the mean $\langle\Delta \eta\rangle$ for $\sqrt{s_{b}}=$ $630 \mathrm{GeV}$ in Fig. 田. At large $\Delta \eta>$ the dijet cross section increases almost by a factor of 3 between the two $\mathrm{cm}$ energies. The strong increase is reflected in a large mean $\lambda$ value: $\lambda=0.65 \pm 0.07$, stronger than expected by theoretical predictions 21 . The exact LO pQCD calculation leads to a falling cross section. The LO BFKL calculation (labelled LLA in Fig. (4) predicts $\lambda=0.45$ for $\alpha_{s}(20 \mathrm{GeV})=0.17$. A complete NLO BFKL calculation is not yet available. Surprisingly, the highest $\lambda=0.6$ is obtained by HERWIG, a LO QCD event generator supplemented by leading log parton showers.

However, it has recently been pointed out 22 that an interpretation of these results is difficult because of differences in the definition of the cross sections between the D0 data and the original Mueller-Navelet proposal. This concerns the reconstruction of the momentum fractions $x_{i}$ using eq. 3 which is only valid for 2-body kinematics and the presence of an upper bound on the momentum transfer $Q^{2}$. These differences to the original proposal can be neglected only in the asymptotic limit at large $s$ and large $\Delta \eta$. At TEVATRON, however, this limit is not reached. Moreover, the requirement of two jets with the same minimum $E_{T}$ is particularly critica 23 , since large logarithms not connected with BFKL effects make an interpretation very difficult.

\subsection{Inclusive Hadronic Cross Section in $\gamma^{*} \gamma^{*}$ Collisions}

By studying the total hadronic cross section $\gamma^{*} \gamma^{*} \rightarrow$ hadrons in $e^{+} e^{-}$collisions, difficulties connected with the hadronic nature of

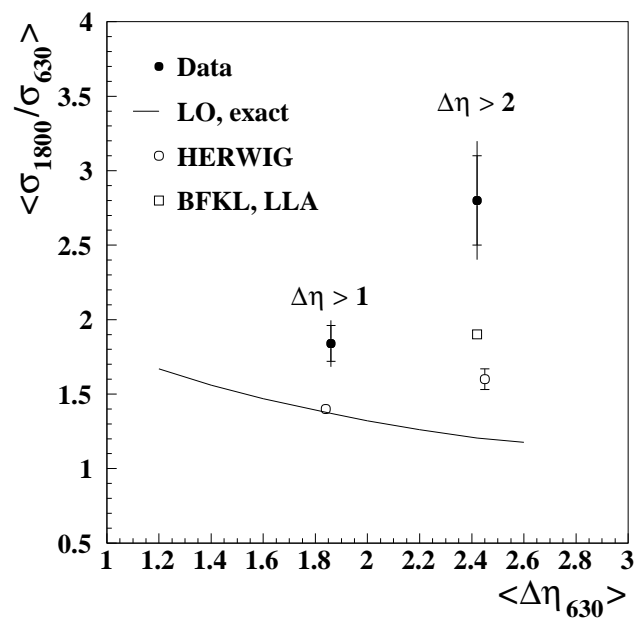

Figure 4. Ratio of the D0 dijet cross section at a cm energy $\sqrt{s}=1800 \mathrm{GeV}$ and $\sqrt{s}=630 \mathrm{GeV}$ for $\Delta \eta>$ 1 and $\Delta \eta>2$ as a function of the mean rapidity difference of the jets at $\sqrt{s}=630 \mathrm{GeV}$.

incoming particles can be naturally avoided. Virtual photons are colourless objects and their virtuality $Q^{2}$ controls the transverse size $\propto 1 / \sqrt{Q^{2}}$ of the hard processes. For $Q^{2} \gg \Lambda_{\mathrm{QCD}}^{2}$ a complete perturbative calculation is possible. For small virtualities $Q_{i}^{2}$ of one of the virtual photons and for large cm energies $W$ of the $\gamma^{*} \gamma^{*}$ system the cross section contains large logarithms of the form:

$$
\sigma_{\gamma^{*} \gamma^{*}} \propto \exp \left(\lambda \ln \frac{W^{2}}{\sqrt{Q_{1}^{2} Q_{2}^{2}}}\right)=\exp (\lambda Y) .
$$

If $Q_{1}^{2} \approx Q_{2}^{2}$, a DGLAP parton evolution between the two photons is suppressed and a resummation of the terms $Y=$ $\ln \left(W^{2} / \sqrt{Q_{1}^{2} Q_{2}^{2}}\right)$ is needed. $\sigma_{\gamma^{*} \gamma^{*}}$ is there-

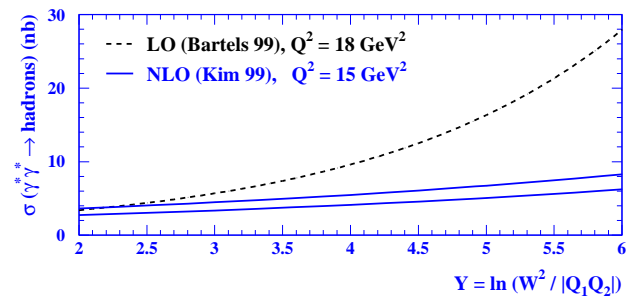

Figure 5. Total hadronic cross section for the reaction $\gamma^{*} \gamma^{*} \rightarrow X$ as a function of $Y$ calculated in a LO and an approximate NLO BFKL calculation.

fore often considered as a golden BFKL signature. An example of a Feynman diagram is shown in Fig. 3c. As can be seen in 


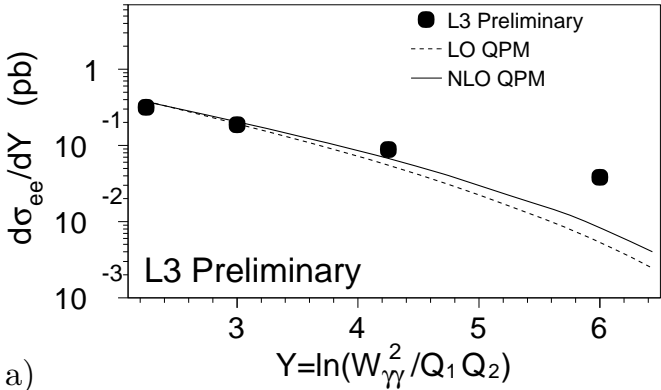

a)

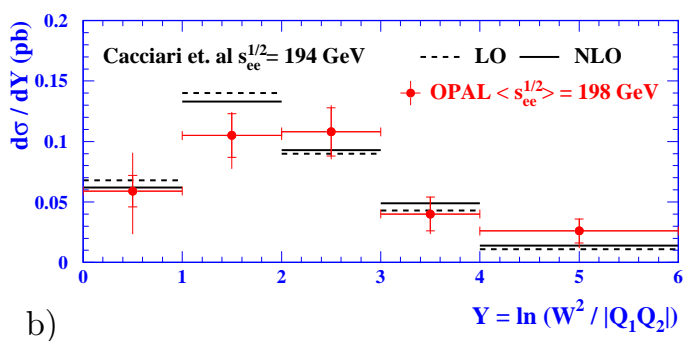

Figure 6. Total hadronic cross section $\gamma^{*} \gamma^{*} \rightarrow X$ as a function of the variable $Y$ a) measured by L3 and b) by OPAL. Overlayed is a LO and NLO fixed order calculation.

Fig.5, in LO a strong increase of $\sigma_{\gamma^{*} \gamma^{*}}$ at high $Y$ is expected 4 . An approximation to a NLO calculation 25 leads to a much suppressed cross section at high $Y$.

In a first measurement of $\sigma_{\gamma^{*} \gamma^{*}}$ in $e^{+} e^{-}$ collisions at $\sqrt{s_{e e}}=91-183 \mathrm{GeV}$ the predicted significant rise towards large $Y$ was observed by L326. However these data have not been corrected for QED radiative effects. In particular, if the kinematics is reconstructed using the scattered electrons, these corrections are substantial. In a recent analysi.27, L3 has presented cross sections corrected for radiative effects using $e^{+} e^{-}$data at a cm energy of $\sqrt{s_{e e}}=189-209 \mathrm{GeV}$. The QED corrections lower the measured uncorrected cross section by $58 \%$ at large $Y$ and, moreover, can be very different for the various contributing QCD subprocesses. The cross section measured in the kinematic region: $4<Q_{i}^{2}<44 \mathrm{GeV}^{2}, W>5 \mathrm{GeV}$, electron energy $E_{i}>40 \mathrm{GeV}$ and the polar electron angle $30<\theta_{i}<66 \mathrm{mrad}$ is shown in Fig. 6a). A fixed order calculation in LO as well as in $\mathrm{NLO}^{28}$ is able to describe the data at low $Y(Y<5)$. However, at the largest $Y(5<Y<7)$, the calculations are below the data by 4 standard deviations. The NLO corrections are relatively small and they only slightly increase the cross section at large $Y$. L3 concludes that "this can indicate the presence of QCD resolved processes or the onset of BFKL dynamics" 27 .

However, this effect has not been confirmed by OPAL29 analysing $e^{+} e^{-}$collisions at $\mathrm{cm}$ energies of $\sqrt{s_{e e}}=189-209$ $\mathrm{GeV}$. The measured cross section $\sigma_{\gamma^{*} \gamma^{*}}$ (for $E_{i}>0.4 E_{b}$, where $E_{b}$ is the beam energy, $34<\theta_{i}<55 \mathrm{mrad}$ and $W>5 \mathrm{GeV}$ ) for an average $Q^{2}=17.9 \mathrm{GeV}^{2}$ is shown in Fig. (6) as a function of $Y$. The exact LO and the NLO calculation reproduce the data well. Also an approximate higher order BFKL calculation 30 imposing energy momentum conservation along the gluon ladder, is in agreement with the data (not shown). Although in the L3 analysis slightly higher $W$ and $Y$ values are reached, the different conclusions which can be drawn from the two analyses is surprising. Some experimental questions in particular the control of the radiative corrections as a potentially large source of systematic uncertainty needs to be rediscussed $]^{g}$ before firm conclusions can be drawn. To unambiguously establish small- $x$ effects in $\gamma^{*} \gamma^{*}$ collisions one probably needs much higher $\mathrm{cm}$ energies, like the one that will be available at TESLA.

\subsection{Inclusive DIS: $F_{2}$}

A classic key measurement to understand the structure of the proton and its dynamical processes is the precise determination of the structure function $F_{2}$ by counting inclusively the lepton scattered off the proton. The HERA measurements 31,32 span about 6 orders of magnitude in $Q^{2}, 0.1 \lesssim$

${ }^{g}$ One question is e.g. to which extent the size of the QED corrections depend on the Born cross section itself. An iterative procedure might be required. 


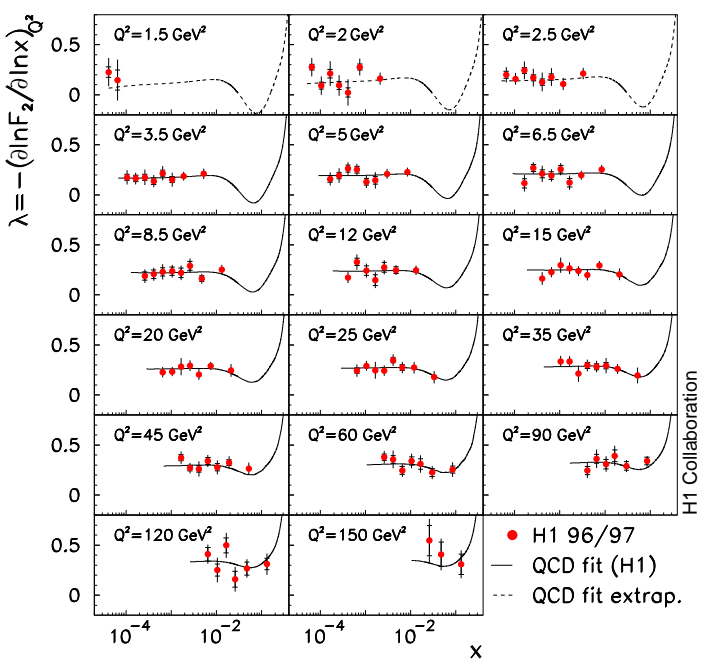

Figure 7. Slope of the proton structure function $\lambda$ as a function of $x$ in bins of $Q^{2}$.

$Q^{2} \lesssim 30000 \mathrm{GeV}^{2}$, and cover the momentum range from the sea quark region at low $x\left(x \gtrsim 10^{-6}\right)$ to the valence quark region at large $x(x \lesssim 0.65)$.

Compared to observables based on the hadronic final state, the measurement of $F_{2}$ has the advantage that it can be directly compared to pQCD calculations. However, because of its inclusive nature, small novel effects on top of the dominating DGLAP parton evolution might be difficult to reveal. Therefore $F_{2}$ has to be precisely pinned down to get a handle on the QCD evolution and to simulanteously constrain the nonperturbative parton density functions. In the most recent $F_{2}$ measurements a precision of $2-4 \%$ in the high statistics region $Q^{2} \lesssim 100 \mathrm{GeV}^{2}$ has been achieved 32 . One of the most important results is that the rise of $F_{2}$ towards small- $x$ :

$$
\sigma_{e p} \propto F_{2} \propto \exp \left(\lambda \ln \frac{W^{2}}{Q^{2}}\right) \approx x^{-\lambda}
$$

expected in the DLL or BFKL (see sec. 2) limit has been observed. However, analyses based on the NLO DGLAP equations give a satisfactory description of the data down to remarkably low $Q^{2}$ and $x$ values 33 34. 35 . In these analyses the quark and gluon densities

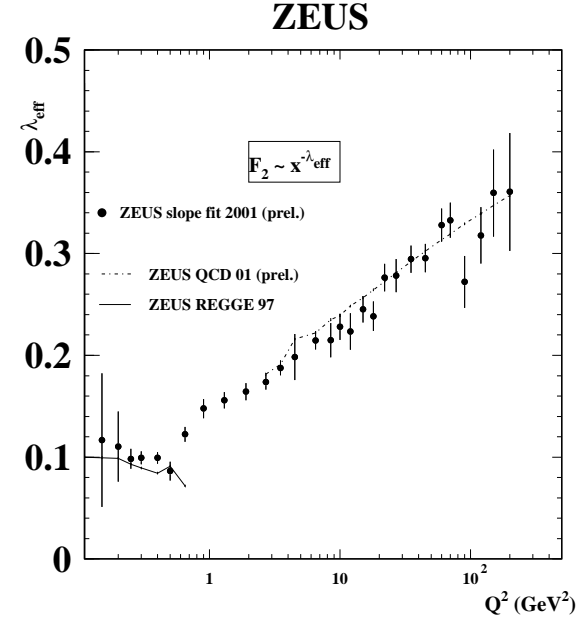

Figure 8. Slope of the incl. total $\gamma^{(*)} \mathrm{p}$ cross section $\lambda$ as a function of $Q^{2}$. Shown is a generalised vector dominance model and a QCD NLO DGLAP fit.

are parameterised at a starting scale and are adjusted such that the data are described. While $F_{2}$ constrains mainly the quark densities, the scaling violation $d F_{2} / d \log Q^{2}$ give access to the gluon density. Whether this proves the correctness of the DGLAP picture or is just due to a large flexibility of the fit, in particular in the parameterisation of the parton density function, can not yet be answered. To improve the sensitivity to small- $x$ QCD effects, the $\mathrm{H} 1$ collaboration 36 has measured the $F_{2}$ slope $\lambda$ (see eq. 50) as a function of $x$ for fixed $Q^{2}$ (see Fig. (7). In this way the strong $Q^{2}$ dependence of $\lambda$ is removed. For fixed $Q^{2}, \lambda$ does not change with decreasing $x$ and the NLO DGLAP fit describes the data. The $Q^{2}$ dependence of $\lambda$ is even more visible in the ZEUS measuremnet shown in Fig. 8 (see also sec. 价. Despite this success it is worth noting that a common feature of the NLO DGLAP fits is that the longitudinal structure function $F_{L}$ becomes negative, which is obviously unphysical. The inclusion of $\ln (1 / x)$ terms is able to cure these problems 4 , 37.38. If one aims for a global description of all available data, the standard NLO PGLAP fit has to be stretched to the edge 39 . In particular, there is a complicated interplay between the small- $x$ DIS data and the high- $x$ inclusive jet cross sections from 


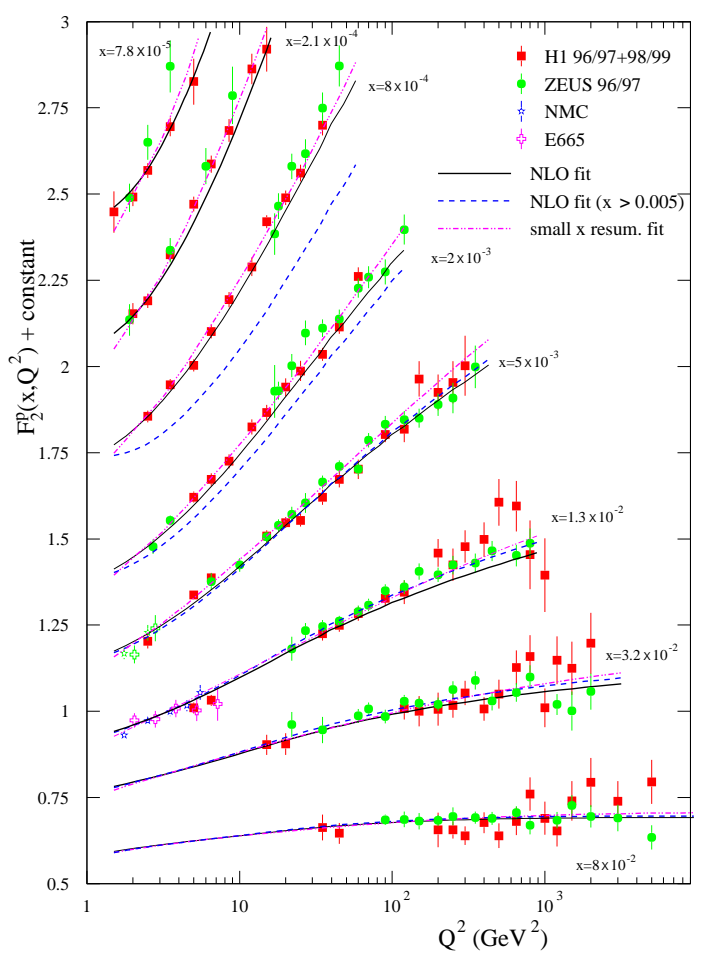

Figure 9. Proton structure function data as a function of $Q^{2}$ at fixed $x$ compared with several different fits within the MRST global fit framework.

TEVATRON 4 41 4 . The jet data prefer a high gluon at large $x$ values $(x>0.1)$ and this significantly influences the small- $x$ gluon via the momentum sum rule. The DIS data prefer a smaller gluon at high- $x$ values. Therefore, some kind of compromise has to be found. Within the present accuracy of the data, this is still possible, but is seems that there is not much freedom left in the NLO DGLAP fit.

Within the framework of the MRST global QCD analysis 34.39 , it has been shown that problems in describing HERA $F_{2}$ data at medium- $x$ values $\left(\approx 10^{-2}\right)$ can be avoided, if the data at small- $x\left(x \lesssim 10^{-3}\right)$ are not considered in the fit or, alternatively, if a small- $x$ resummation obtained by solving the running coupling BFKL equation is included 3742. This is demonstrated in Fig. 9 m where the standard NLO DGLAP fit gives a bad description of the data at $x=1.3 \cdot 10^{-2}$ and $100 \lesssim Q^{2} \lesssim 1000 \mathrm{GeV}^{2}$ while a fit only in-

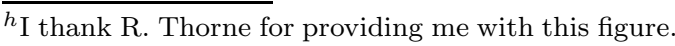

cluding data with $x \gtrsim 5 \cdot 10^{-3}$ is much better. This fit, however, does not describe the small- $x$ data. When small- $x$ resummation is included the data can be described over the whole kinematic domain.

In conclusion, the NLO DGLAP fit describes DIS data to surprisingly small- $x$ and $Q^{2}$ values. However, there are some indications that the flexibility in the input parameters is stretched to the edge, in particular, if the new inclusive high $E_{T}$ jet data from TEVATRON are included. Fits including small- $x$ resummation lead to an improved description of the data and a better convergence of $F_{L}$ when going from LO to NLO.

\subsection{Heavy Quark Production}

The production of heavy quarks $Q$ in DIS is directly sensitive to the gluon content of the proton, since in LO the process $\gamma g \rightarrow Q \bar{Q}$ dominates. Heavy quark cross sections are therefore an excellent testing ground for the dynamics of the parton evolution. The inclusive $D^{*}$ meson cross section has recently been measured by $\mathrm{H}_{143}$ in the range $1<$ $Q^{2}<100 \mathrm{GeV}^{2}, 0.05<y<0.7, p_{t}^{D^{*}}>$ $1.5 \mathrm{GeV}$ and $\left|\eta^{D^{*}}\right|<1.5$ to be: $\sigma_{v i s}=$ $8.5 \pm 0.42$ (stat) $)_{-0}^{+1.02}$ (syst) \pm 0.65 (model) nb. A NLO calculation 44 based on the heavy quark matrix elements, DGLAP parton evolution and the Peterson fragmentation function 45 only predicts $\sigma_{v i s}=5.1-7 \mathrm{nb}$ depending on the exact value of the charm quark mass $m_{c}$ and the Peterson fragmentation parameter $\epsilon$. The most significant difference between data and NLO is observed towards the proton direction, i.e. at large $\eta$. Similar results have been obtained by ZEUS46. A possible explanation that this effect is due to neglecting the colour force between the charm quark and the proton remnant 47 is not large enough to explain this discrepancy. The CCFM prediction, based on the off-shell matrix elements and on an unintegrated gluon density obtained from a $F_{2}$ fit, has been calculated using CASCADE 16 and agrees with the data. 


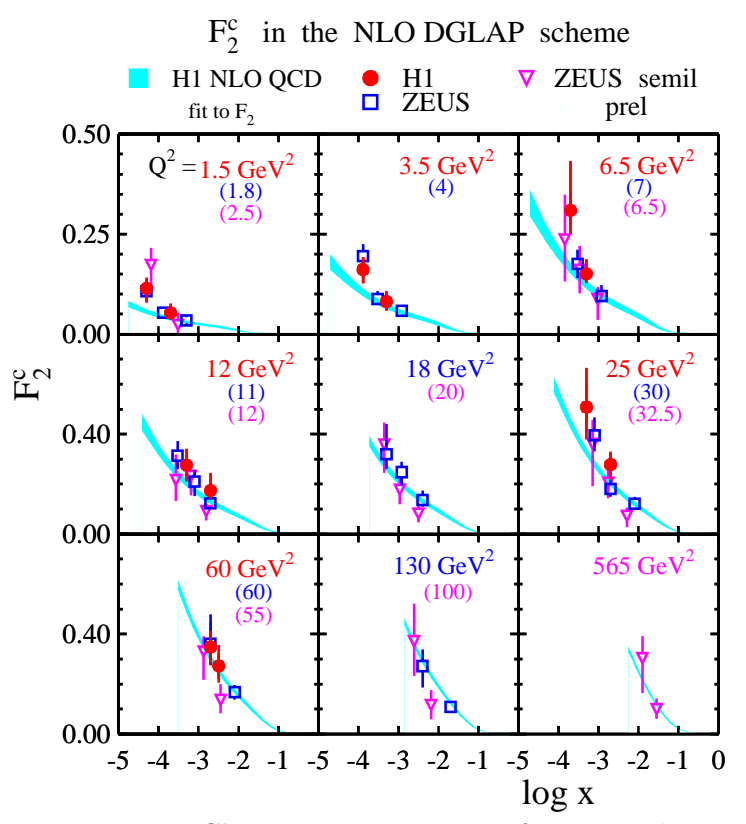

Figure 10. Charm proton structure function $F_{2}^{c}$ as a function of $\log x$ in $Q^{2}$ bins.

The visible cross section can be converted to the semi-inclusive charm structure function $F_{2}^{c}$ which is easier to compare to theory predictions. However, the extraction of $F_{2}^{c}$ faces the problem that only part of the total cross section (typically $30 \%$ ) can be measured in the detector. This quantity is therefore significantly biased by the theoretical model used to determine the experimental acceptance (up to $20 \%$ at the smallest $x$ ). $F_{2}^{c}$ extracted in the NLO DGLAP scheme is shown in Fig. 10. A steep rise towards small- $x$ and a clear dependence of the slope $\lambda$ on $Q^{2}$ is observed. In particular, in the low $Q^{2}$ region this rise tends to be steeper than predicted by the NLO DGLAP calculation?. At small$x, F_{2}^{c}$ grows at the same rate as the fully inclusive structure function $F_{2}$ as is expected, since both processes are initiated by gluons.

This year $\mathrm{H} 1$ has reported 48 on the first measurement of beauty production in DIS in the region $2<Q^{2}<100 \mathrm{GeV}^{2}, 0.05<y<$ 0.7 for muon polar angles $35^{\circ}<\theta<130^{\circ}$ and transverse momenta $p_{T}>2 \mathrm{GeV}$. The

\footnotetext{
${ }^{i}$ This is true when compared to the H1 NLO DGLAP fit. In the ZEUS fit a slightly larger $F_{2}^{c}$ error band is obtained making the above conclusion less clear.
}

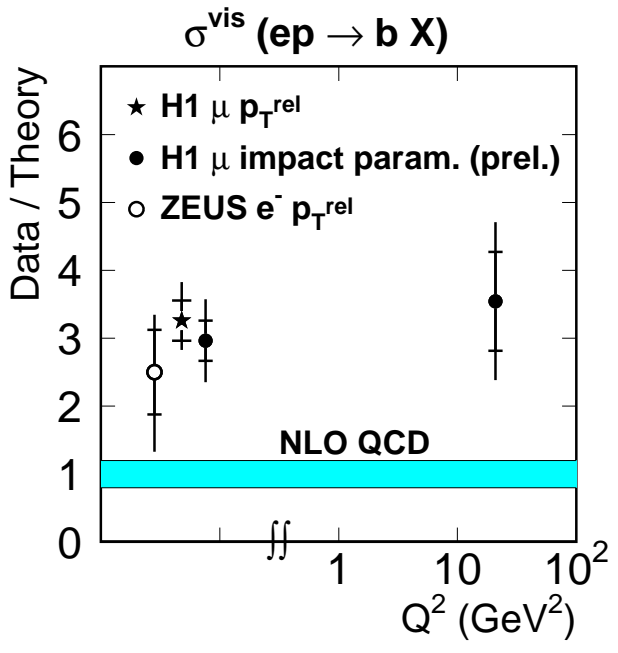

Figure 11. Ratio of the measured to the calculated total beauty production cross section in ep collisions.

beauty cross section is derived by determining the fraction of beauty events decaying semi-leptonically to muons using the transverse momentum of the muon with respect to the jet axis $\left(P_{T, r e l}\right)$ and by exploiting the finite lifetime of b-mesons by measuring the distance of closest approach of the muon track with respect to the primary event vertex (impact parameter). The measured cross section $\sigma_{e p \rightarrow b X \rightarrow \mu X}=39 \pm 8$ (stat) \pm 10 (syst)pb is significantly above the NLO prediction 44 of $\sigma=11 \pm 2 \mathrm{pb}$. In the calculation, a beauty mass $m_{b}=4.75 \mathrm{GeV}$, a renormalisation and factorisation scale $\mu=$ $\sqrt{Q^{2}+4 m_{b}^{2}}$ and a Peterson fragmentation parameter $\epsilon=0.0033$ are used. The LO cross section is only $\sigma=9 \mathrm{pb}$. The CASCADE program based on the CCFM equation predicts $\sigma=15 \mathrm{pb}$. A similar excess has been reported in quasi-real photonproton collisions by $\mathrm{H} 149$, where the cross section for $b \bar{b}$ production for $Q^{2}<1 \mathrm{GeV}^{2}$ is measured. A cross section value larger than the theory prediction js also reported by the ZEUS collaboration 50 in the semi-leptonic electron channel. The $b \bar{b}$ cross section for $Q^{2}<1 \mathrm{GeV}^{2}, 0.2<y<0.8, p_{t, b}>5 \mathrm{GeV}$ and $\left|\eta_{b}\right|<2$ is: $\sigma_{e p \rightarrow e b \bar{b} X}=1.6_{-0.75}^{+0.54} \mathrm{nb}$. The NLO prediction is only $0.64 \mathrm{nb}$. 


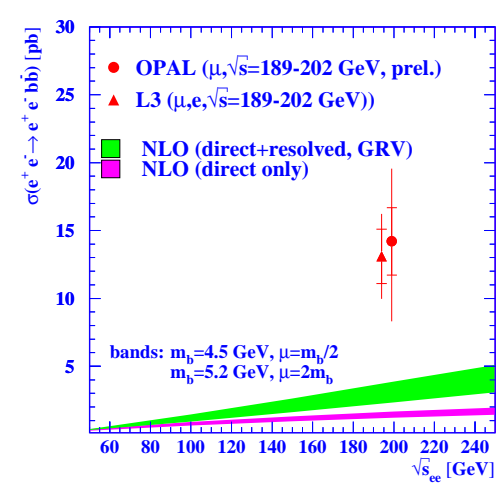

Figure 12. Total beauty production cross section in $\gamma \gamma$ collisions as a function of the cm energy $\sqrt{s_{e e}}$.

A comparison between data and theory for the various measurements is shown in Fig. 11. For all measurements a higher cross section with respect to the NLO predictions is measured. However, the total cross section measurement is affected by large extrapolation factors calculated using LO MC simulation programs. For instance, jets with $E_{T}>6 \mathrm{GeV}$ have to be selected to calculate $P_{T, \text { rel }}$. In the quoted total cross section they are not included. Only $42.9 \%$ of the cress section is really measured in the detector 49 . Moreover, requiring two jets with equal $E_{T}$ is problematic 23 . The extrapolation includes large model uncertainties50.51 (up to 30\%). It is interesting that the ZEUS measurement, where also the beauty cross section including the jet requirement is quoted, agrees well with the CASCADE result and with LO QCD MC simulations, if heavy quark excitation processes possibly mimicking part of the NNLO corrections 47 are included.

The increase of the $\mathrm{cm}$ energy of LEP to $\sqrt{s_{e e}} \approx 200 \mathrm{GeV}$ has also made possible the first observation of beauty production in $\gamma^{*} \gamma^{*}$ collisions. Here also an excess of the measured beauty cross_section over the theory prediction is found52 (see Fig. 12). The NLO prediction agrees with the measured charm cross sections, but in the case of beauty it underestimates the cross section by about two standard deviations. Since many of the phenomenological challenges are similar to $e p$ collisions, e.g. the description of

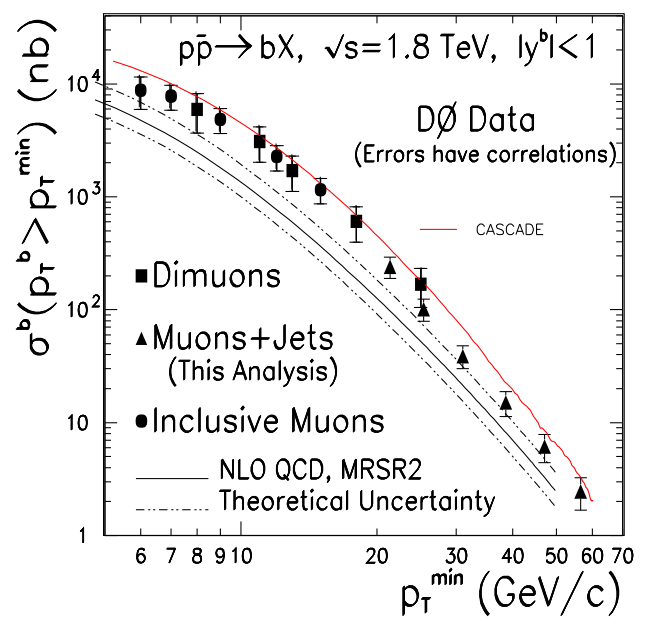

Figure 13. Beauty production cross section for different minimal values of the transverse beauty momentum in $p \bar{p}$ collisions at $\sqrt{s}=1800 \mathrm{GeV}$.

heavy quarks near the production threshold or the treatment of the hadronic structure of the photon, this indicates that the excess of beauty events at HERA is not accidental, but might have a deeper reason.

Also in $p \bar{p}$ collision a higher beauty cross section has been measured 53 than expected by NLO calculations 54 . In general, the observed excess grows towards large rapidities, i.e. towards the proton remnants. One of the measurements performed in the central rapidity region is shown in Fig. 13 over a wide minimum transverse momentum range $p_{T, b}^{\min }$. Shown is the b-meson cross section (incl. muon) as well as a jet cross section where the jets contain a b-tag (muon+jet). For the last observable the treatment of soft and collinear divergencies is safer in the theoretical calculation 55 . For both observables the data lie higher than the theory prediction. Even so, the agreement with the theory is improved in the latter case in particular at high $p_{T, b}^{\min }$, a discrepancy still remains with respect to the central result.

A better agreement with the data is found using CASCADE based on the CCFM equations 56 (see Fig. 13). This approach uses LO off-shell matrix elements for heavy quarks and the scale is set to $\mu^{2}=m_{b}^{2}+p_{T, b}^{2}$. In ad- 
dition, the unintegrated gluon density, which has been obtained from an input parameterisation using only two parameters adjusted to HERA data, enters the calculation 5 . Note, that in a typical NLO DGLAP fit much more free parameters are used to parameterise the parton densities. In view of the few input parameters only fixed by DIS data the agreement of the CASCADE calculation with the data is quite remarkable. A similar success in describing beauty production in $p \bar{p}$ collisions has been obtained using $k_{T}$ factorisation, an unintegrated parton distribution and a BFKL vertex for the $q \bar{q}$-vertex $\bar{Z}$.

Whether the higher beauty cross sections seen in various processes really can be attributed to the necessity to modify the parton evolution at small-x $x$ or whether it is just due to other complications like the resummation of large logarithms in the presence of two or more hard scales or problems connected to the modelling of the fragmentation cannot be answered today. The larger amount of data which will be available after the HERA and TEVATRON luminosity upgrades together with the improved efficiency of the upgraded detectors will allow further improvements in the experimental precision and to extend the measurement towards larger $p_{T}$ and larger $Q^{2}$. This might allow possible solutions to this problem to be disentangled.

\subsection{Forward Jet and Particle Production in DIS}

The classical signature designed to enhance BFKL effects in DIS is the production of "forward jets" 58 characterised by $k_{T}^{2} \approx Q^{2}$ and $x_{\text {jet }}=E_{\text {jet }} / E_{p}$ as large and $x$ as small as kinematically possible (see Fig.3 3 d) $)$. The first requirement suppresses the $k_{T}$ ordered

\footnotetext{
${ }^{j}$ At the TEVATRON cm energy, the gluon-gluon fusion is the dominant production mechanism.

${ }^{k}$ Note that the $x$ values in some of the observables are not really small. Moreover, the work to include finite $z$ terms in CASCADE is still in progress.

${ }^{l} E_{\text {jet }}\left(E_{p}\right)$ denotes the energy of the forward jet (proton) in the laboratory system.
}

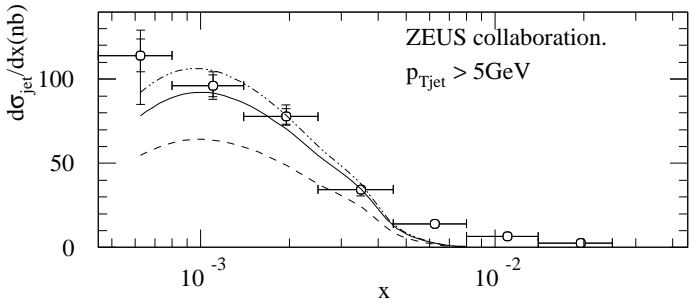

Figure 14. Forward jet DIS cross section as function of $x$. Approx. NLO BFKL calculations for different scales and infra-red cut-offs are shown as lines.

DGLAP evolution. When asking for large $x_{\text {jet }} / x$, the forward jet is separated by a large rapidity interval from the struck quark such that the phase space for parton emissions between the two is amplified. In this case the $\alpha_{s} \ln \left(x_{\text {jet }} / x\right)$ terms are expected to become so large that their resummation leads to a sizable increase of the forward jet cross-section:

$$
\sigma_{B F K L} \propto \exp \left(\lambda \ln \frac{x_{j e t}}{x}\right) .
$$

A fast rise of the cross-section for forward jets with decreasing $x$ has indeed been seen in the data59. A LO BFKL calculation 60 rises steeper than the data. A more recent calculation emulating some of the expected NLO effects 61 is in much better agreement (see Fig.14). Also CASCADE based on the CCFM equations reproduces the data17.

The good description of the forward jet cross section can be taken as an indication of the need for the BFKL resummation in an extreme phase space, where small- $x$ effects are expected to be important. However, other interpretations are possible. For instance, a calculation including direct and resolved photon interaction also gives a good description of the data62 63. Kramer and Pötter argue that the success of their NLO calculation can be taken as a strong indication that a direct $\mathcal{O}\left(\alpha_{s}^{3}\right)$ calculation, i.e. without the need to introduce a virtual photon structure, would also describe the data. In this case the need for a BFKL resummation is just not strong enough at HERA to be unambiguously distinguished from an exact fixed order calculation. However, a recent H1 analysis 64 sys- 
tematically investigating the phase space regions from a simple inclusive jet cross section to the forward jet regime shows that neither a direct NLO calculation nor the NLO calculation including resolved photons describes the data in the whole phase space. While e.g. the forward jet cross section is described, the calculation overshoots the simple single inclusive jet cross section in the backward region.

In conclusion, despite the wealth of jet data at small- $x$ which became recently available a consistent physical interpretation has not been achieved.

A complementary approach to gain insights in the parton evolution is the production of particles at $E_{T} 65$. Charged or neutral particles at high $E_{T}$ are well correlated to parton activity. Their production rate can be directly predicted by analytic calculations folded with proper fragmentation functions. The measured $\pi^{0}$ cross section exhibits the same rise towards small- $x$ as the inclusive one, i.e. the ratio $R_{\pi}(x)$ is constant (see Fig. 15)66. At $2<Q^{2}<4.5 \mathrm{GeV}^{2}$ approximately $0.2 \%$ of the DIS events contain a forward $\pi^{0}$ with $E_{T}>2.5 \mathrm{GeV}$. At $15<Q^{2}<70 \mathrm{GeV}^{2}$, this ratio rises to $0.5 \%$. The MC based on LO QCD and parton showers (labelled LEPTO) cannot describe this behaviour, while a BFKL calculation approximating some of the NLO effects by requiring energy-momentum conservation and allowing for a running coupling describes the data 61 .

\section{Inclusive and Diffractitive DIS, Transition from Soft to Hard}

The constant (with respect to the inclusive cross section) probability to emit hard particles in the forward region can be juxtaposed to the probability to emit no particle at all. In approximately $5-10 \%$ of all DIS events no particle is measured in a large rapidity region 67. The latter class of events are called "diffractive" rate indicates a deep connection between the

$\bar{m}$ In the analogy of the diffraction of light, see 68 and refs. therein.

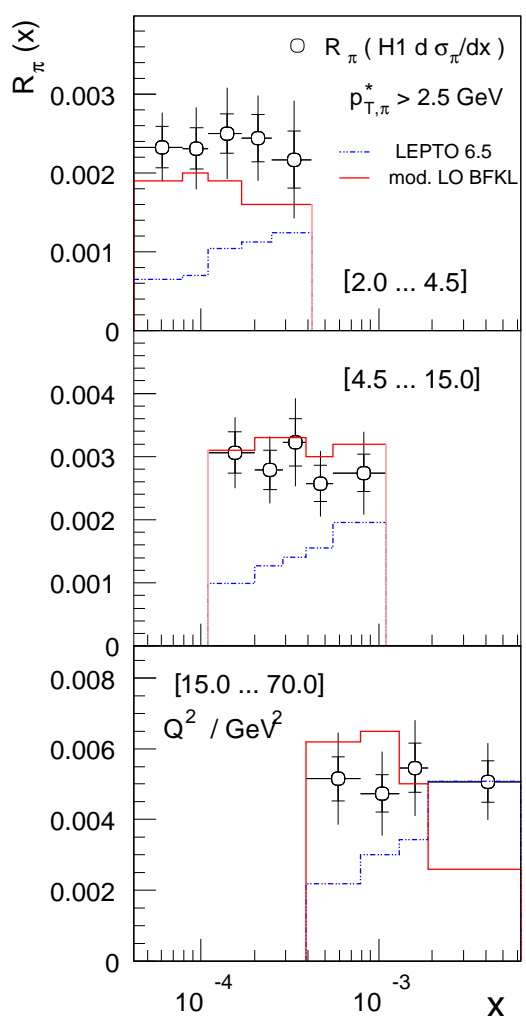

Figure 15. Rate of forward $\pi^{0}$ to the inclusive cross section as a function of $x$.

production mechanism of rapidity gap events and the parton dynamics at small- $x$.

The interaction responsible for a large rapidity gap can be viewed as the exchange of a colour singlet decomposing the hadronic final state into a system $X$ and $Y: e p \rightarrow e X Y$ (see Fig. 16). The kinematic of the process can be described by the longitudinal momentum fraction $\xi$ of the colourless exchange with respect to the incoming proton ${ }^{m}$ and, in analogy to Bjorken- $x$ in the inclusive case, by the longitudinal momentum fraction $\beta$ that is carried by the quark struck by the photon (see Fig. 16). The ratiof

$$
\rho^{D(3)}=M_{X}^{2} \frac{d \sigma_{\gamma^{*} p \rightarrow X Y} / d M_{X}^{2}}{\sigma_{\gamma^{*} p \rightarrow X}}
$$

is shown in Fig.17 as a function of the $\gamma^{*} p$

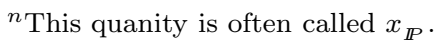

${ }^{\circ}$ The diffractive cross section is in this analysis integrated over $M_{Y}<1.6 \mathrm{GeV}$ and $t<1 \mathrm{GeV}^{2}$, where $t$ is the squared momentum transfer from the proton to the system $\mathrm{Y}$.
} 


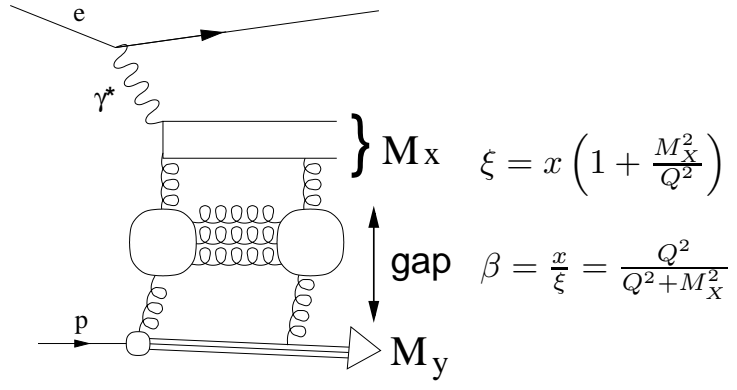

Figure 16. Sketch of a diffractive scattering at HERA.

$\mathrm{cm}$ energy 69 . The ratio $\rho^{D(3)}$ is relatively flat over the full phase space. Only at low $\beta$ a tendancy to rise is observed. The constant rate as a function of the cm energy has already been observed in the first HERA data 0 , but it is still rather surprising, when one tries to understand its physical meaning. In the simplest approach 7 the colour singlet exchange can be modelled by the exchange of two "gluons" 2 . If the "gluons" are interpreted as partons, i.e. pQCD is applicable because a hard scale is involved in the process, then at small- $x$ the inclusive cross section is expected to rise like $\sigma \propto x^{-\lambda}$ while the diffractive cross section should rise like $\sigma \propto x^{-2 \lambda}$. In this case the rapidity gap contribution would significantly increase towards small- $x$. If the colour singlet exchange is soft, the increase of the diffractive cross section should be closely related to soft hadron hadron and $\gamma p$ collisions where $\sigma \propto W^{0.08}$ and therefore $\sigma \propto(1 / x)^{0.16}$ is expected. In this case the rapidity gap contribution in the DIS sample would decrease.

The constant behaviour of $\rho^{D(3)}$ indicates that the production mechanism of rapidity gap events contains soft and hard pieces. It is remarkable that their interplay leads to the same energy dependence of the cross section as in the inclusive case.

For inclusive $\gamma^{*} p$ collisions, $Q^{2}$ provides the hard scale to discriminate soft and hard processes. The energy behaviour of the in-

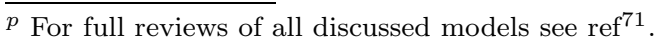

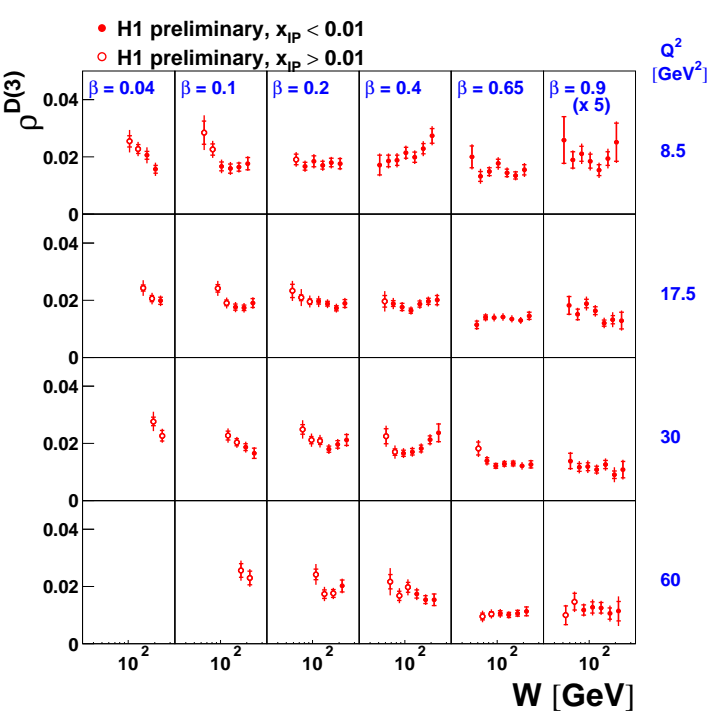

Figure 17. Ratio of the diffractive to the inclusive DIS cross section as function of the $\gamma^{*} p$ centre of mass energy $W$ in bins of $Q^{2}$ and $\beta$.

clusive cross section (see eq. [5) is shown in Fig.8. Recently, ZEUS has achieved a remarkable precision on the inclusive cross section measurements at very low $Q^{2}$ thanks to dedicated detectors close to the beam pipe measuring electrons scattered at small angles 74 . For $Q^{2} \lesssim 0.8 \mathrm{GeV}^{2}$, a constant slope $\lambda \approx 0.1$ is found. This value is consistent with the value $(\lambda=0.08)$ needed to describe the total hadron hadron cross section at high energies 53 . The same value also describes the energy behaviour of the scattering of real photons on protons 76 . A generalised vector dominance mode 75 describes the data. For higher $Q^{2}$ values, $\lambda$ linearly rises with $\log Q^{2}$ which is well described by pQCD. The transition from the soft to the hard regime seems to be surprisingly sharp $\left(0.5 \lesssim Q^{2} \lesssim 1 \mathrm{GeV}^{2}\right)$. Does this indicate that there are really two different physical regimes, the long-distance, non-perturbative region, where only Regge theory can be applied, and the short-distance, pQCD region describing the interaction of quarks and gluons ? Or is a unified description based on small- $x$ phenomena possible?

The physical understanding of the transition region can be made more intuitive in 
the proton rest frame rather than in the DIS frame, where the proton has infinite momentum. In this frame the (virtual) photon splits into a $q \bar{q}$ pair and develops a hadronic structure by radiating gluons long before interacting with the proton. The timescale of this fluctuation is $\tau \propto 1 /\left(x m_{p}\right)$. At small- $x$, the lifetime $\tau$ of the hadronic system is long, e.g. at $x=10^{-3}, c \tau=200 \mathrm{fm}$. The $q \bar{q}$ pair can be viewed as a colour dipole described by a wave function $\Psi_{T, L}$ depending on the lopgitudinal and transverse photon polarisation 7 . It can be calculated within QED. The $\gamma^{*} p$ cross section can be written as:

$$
\sigma\left(x, Q^{2}\right)=\int d^{2} r d z\left|\Psi_{T, L}\right|^{2} \hat{\sigma}(x, r)
$$

where $r$ is the transverse separation of the $q \bar{q}$ dipole and $z$ is the photon momentum fraction carried by the quark. By the Heisenberg principle $r$ is closely related to the transverse quark momentum via $r \propto 1 / k_{T}$. For small $k_{T}$, i.e. large dipoles, the colour field is large and it strongly interacts with the proton. The dipole behaves as a hadron in soft collisions. For large $k_{T}$, i.e. small dipoles, the colour field is effectively screened and the proton is "transparent" to the dipole (colour transparency). $\hat{\sigma}(x, r)$ describes the interaction of the $q \bar{q}$ dipole with the proton.

The following phenomenological form has been proposed 79 for $\hat{\sigma}(x, r)$ :

$\sigma_{0}\left(1-\exp \frac{-r^{2}}{4 R_{0}^{2}(x)}\right)$ with $R_{0}^{2}(x)=\left(\frac{x}{x_{0}}\right)^{-\lambda}$

where $\sigma_{0}=23 \mathrm{mb}, x_{0}=3 \cdot 10^{-4}$ and $\lambda=0.29$ are determined by a fit to inclusive DIS data for $x<0.01$ As an example, the dipole cross section is shown at $Q^{2}=10 \mathrm{GeV}^{2}$ in Fig. 19. For small $r$, the dipole cross section steeply rises $\hat{\sigma}(x, r) \propto r^{2} x^{-\lambda}$ as expected by pQCD. Towards large $r$ the cross section is assumed to saturate $\hat{\sigma}(x, r) \propto \sigma_{0}$. It is interesting that the integrand of equation 7 shown

${ }^{q}$ The radius $R_{0}$ has the units $1 / \mathrm{GeV}$. These numbers are quoted for a model with no charm quarks. Including charm quarks leads to larger values.

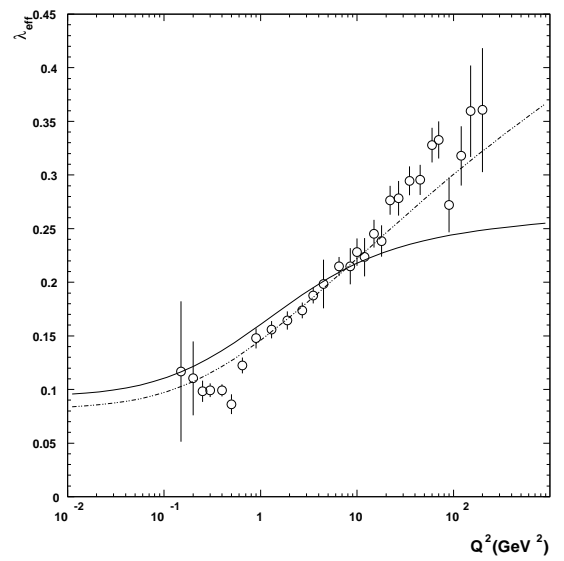

Figure 18. Slope of the inclusive total $\gamma \mathrm{p}$ cross section $\lambda$ as a function of $Q^{2}$. Shown as lines are fits to the Golec-Biernat/Wüsthoff model with and without QCD evolution.

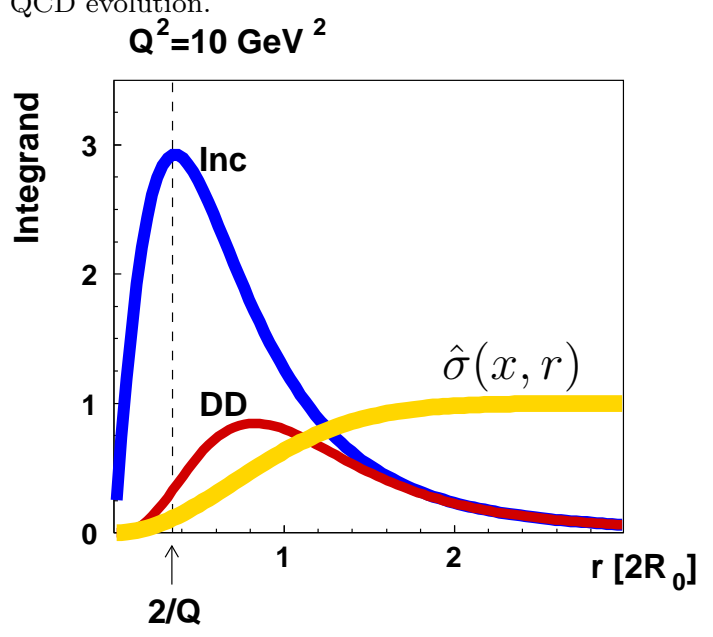

Figure 19. The integrand in equation 6 for inclusive (Inc) and diffractive (DD) scattering and the dipole cross section $\hat{\sigma}(x, r)$.

in Fig. 19 peaks around $2 / Q$ making the close relation between the photon virtuality and the dipole size clear.

With the assumed form of the fitted dipole cross section the inclusive DIS data can be successfully described over a wide kinematic range (see Fig. 18b). The additional incorporation of DGLAP evolution $\$ 1$ is needed to describe the data at large $Q^{2}$. However, it can not fully account for the steep

$r$ To do that, $1 / R_{0}^{2}$ is replaced by $4 \pi^{2} \alpha_{s}\left(\mu^{2}\right) x g\left(x, \mu^{2}\right) /\left(3 \sigma_{0}\right)$ where $\mu^{2}=C / r^{2}+\mu_{0}^{2}$, $x g\left(x, \mu^{2}\right)=A_{g} x^{-\lambda_{g}}$ and $C, A_{g}, \lambda_{g}$ and $\mu_{0}^{2}$ are additional fit parameters. 
rise of $F_{2}$ at large $Q^{2}$. The transition around $Q^{2} \approx 0.5 \mathrm{GeV}^{2}$ seems to be smoother than found in the data. In view of problems to describe some of the recent diffractive data 82 it is questionable, if the DGLAP is the correct evolution to be applied in this context.

The diffractive cross section is obtained by modifying equation 6 for inclusive DIS 7 :

$$
\sigma^{D D}\left(x, Q^{2}\right)=\int d^{2} r d z\left|\Psi_{T, L}\right|^{2} \hat{\sigma}^{2}(x, r)
$$

By analysing only the leading contribution a ratio $\sigma^{D D} / \sigma^{i n c} \propto 1 / \ln \left(Q^{2} R_{0}^{2}(x)\right)$ is found which is only slowly varying with $x$ and $Q^{2}$ and correctly reproduces the data $B$ when in the dipole also $q \bar{q} g$ states are included. Fig. 19 shows that the integrand of eq. 8 contains a significantly higher contribution of large dipole sizes at the same $Q^{2}$ than found in the inclusive case. This makes both the soft and the hard nature of the diffractive production mechanism evident. By assuming a certain interaction of the colour dipole with the proton based on the inclusive DIS data at small-x the Golec-Biernat/Wüsthoff model predicts the diffractive cross section. This underlines the deep connection between diffraction and the small- $x$ parton dynamics which we start to understand better and better. In the leading logarithm approximation the colour dipole formulation and the $k_{T}$ factorisation give an equivalent description of hard processes at high energy 7 .

\section{Instanton Production in DIS}

In $\mathrm{QCD}$, anomalous non-perturbative processes are expected which violate classical laws such as the conservation of chirality. Instanton 83 , non-perturbative fluctuations of the gluon field, represent tunnelling transitions between topologically inequivalent vacua. DIS offers a unique possibility to discover QCD instantons.

In DIS instanton induced processes $(I)$ are dominantly produced in a quark gluon fusion process (see Fig.20) 84 85. The virtuality $Q^{\prime 2}$ of the quark $q^{\prime}$, originating from a photon

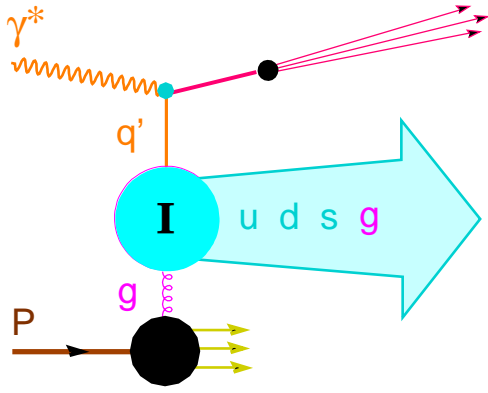

Figure 20. Sketch of the production of instanton induced events in DIS.

splitting into a $q \bar{q}$ pair in the $I$-background, provides a generic hard scale naturally limiting the $I$-size $\rho$ and makes a quantitative prediction of the cross-section possible 486 . The expected cross section for the kinematical region $x>10^{-3}$ and $0.1<y<0.9$ is of the order of $10-100 \mathrm{pb}$.88. This cross section is sizeable, but still much lower than the inclusive DIS cross section. The expected characteristics of the hadronic final state of $I$ processes, which can be simulated using the QCDINS 89 program, can be exploited to improve the signal to noise ration. Besides the current jet, a large number of particles of all kinematically allowed flavours is produced at high $E_{T}$ in a narrow band of two rapidity units. Since the Instanton decays isotropically, a large sphericity of the particles in the $I$-rest system is expected.

The $\mathrm{H} 1$ collaboration has recently performed 91 the first dedicated search for QCD I-induced processes using six hadronic final state $I$-sensitive observables in the kinematic range $x>10^{-3}, 0.1<y<0.6$ and $\theta_{e l}>156^{\circ}$, where $\theta_{e l}$ is the polar angle of the scattered electron's. As an example the sphericity distribution is shown in Fig. 21a for the inclusive DIS sample. Before cuts to enhance the $I$-fraction, the data are well described by pQCD models. The expected instanton sample is about 2-3 orders of magnitude smaller and the events are expected to be more spherical. After cuts the background is suppressed by about a factor of

$\overline{{ }^{s} \text { For an } I \text {-analysis based on existing data see } 92 .}$ 


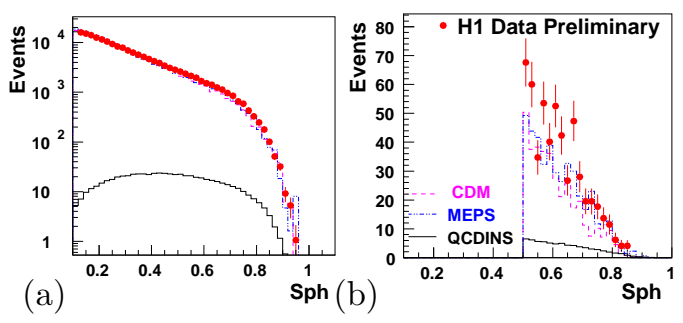

Figure 21. Sphericity for normal and I-DIS without (a) and with (b) cuts to enhance the $I$-signal.

1000. 549 events are selected in the data, while only $363_{-26}^{+22}$ and $435_{-22}^{+36}$ events are expected by two different pQCD models. The size of the excess is, however, about the same as the difference in the pQCD background models. The sphericity distribution of these events is shown in Fig.21 b. In four out of the six $I$-sensitive observables a qualitative similarity between the difference of the data and the pQCD models and the expected $I$ shape is observed. The two remaining observables do not particularly support the hypothesis that the observed excess can be explained by I-processes as currently implemented in QCDINS. However, recently it has been pointed out that the shape of these two particular observables is affected by large theoretical uncertainties due to missing cuts in the experimental analysis 93 . The data limit the allowed $I$-cross section to about $100 \mathrm{pb}$. A steep rise of the I-cross section towards large instanton sizes, as would be obtained from a naive extrapolation of $I$-perturbation theory, is excluded by the data. The absence of such a steep rise is in agreement with lattice simulations of zero flavour, 86,87 .

\section{Conclusions}

The strong cross section increase expected in LO BFKL for various processes has not been observed. Thanks to a huge theoretical effort significant progress has been recently made. The BFKL NLO corrections have been calculated and additional resummations have been worked out. The results are in qualitative agreement with the data. However, no clear and unmistakable evidence for the need for small- $x$ effects beyond the standard resum- mation of leading logarithms using collinear factorisation has been reported so far. Nevertheless there are strong indications that small- $x$ effects play already at the present energies a certain role and that they describe high energy collisions in a more coherent way. First encouraging results using angular ordering as a key unifying principle have been obtained. This allows more precise QCD predictions for future colliders to be made.

The increased precision of the HERA measurements reveals more and more the intriguing connection between soft and hard physics. In the understanding of soft phenomena the correct description of diffraction plays a key role. Here, the deep connection to small- $x$ parton dynamics becomes clearer and clearer and more is to be learned in the near future. The final aim is to get better insights to the confinement of quark and gluons in hadrons. The pioneering experimental and phenomenolgical work to discover QCD instanton-induced processes might add important complementary information in our understanding of non-perturbative phenomena. It seems possible to establish such an important non-perturbative effect at HERA, although no clear experimental evidence has been found so far.

\section{Acknowledgements}

It is a pleasure to thank my colleagues from the H1 and ZEUS collaborations for providing me with the latest data and information. I would like to thank J. Bartels, V. Chiochia, C. Golec-Biernat, G. Iaccobucci, H. Jung, A. Martin, R. Nisius, G. Salam, F. Schrempp, R. Thorne and J. Whitmore for invaluable help in preparing this talk or for commenting on the manuscript.

\section{Questions:}

\section{G. Altarelli, CERN:}

The beautiful HERA data on small- $x$ structure functions pose a clear problem: Why do the NLO QCD evolution equations work so 
well in spite of the fact that the BFKL corrections could be large. You have mentioned the Salam, Ciafaloni, Colferai proposed solution to this problem, but nobody has so far shown that their theory fits the data well. Myself, Ball and Forte we have developed an alternative approach, less predictive, but more model independent (in our opinion) which we have proven to fit the data (we can accommodate but not predict the smallness of the BFKL corrections). Until the understanding of totally inclusive structure functions is not more satisfactory, the theoretical foundations of many BFKL signals in non inclusive distributions is quite shaky.

\section{Answer:}

I agree that the $F_{2}$ data can be interpreted in the theoretically cleanest way, since the uncertainties are usually much smaller than for less inclusive observables. I have pointed out that many open questions remain in the understanding of $F_{2}$ and I have stressed the importance of even more precise data. Since $F_{2}$ is a very inclusive quantity, precision is the key issue. However, less inclusive observables allow to look at new effects in a more direct way and therefore might be more promising. At the end of the day, it will be important to have a consistent theory of strong interactions to describe inclusive and less inclusive measurements in many different processes.

\section{P. Nason, INFN Milano:}

I have a remark with regard to the CCFM Monte Carlo predictions. Monte Carlo programs are practical implementation of theoretical ideas and calculations, and at the end they should match precise analytical calculations. For example, attempts to explain the beauty cross section by small- $x$ resummations, once $\mathcal{O}\left(\alpha_{s}^{2}\right)$ and $\mathcal{O}\left(\alpha_{s}^{3}\right)$ corrections are correctly implemented, failed (until now) to explain the data. Thus, the inaccuracy of the Monte Carlo (that certainly does not implement the $\mathcal{O}\left(\alpha_{s}^{3}\right)$ correctly) should be addressed before claims are made of solving phenomenological problems.

Answer:

The CASCADE MC is an implementation of the CCFM equation. It seems to be adequate, since in all proposed CCFM solutions the correct treatment of the kinematics turned out to be important. Although it is based on LO, part of the higher orders are resummed. In particular when $k_{T}$ factorisation is used, it is difficult to say if, e.g. a $\mathcal{O}\left(\alpha_{s}^{2}\right)$ calculation based on the collinear factorisation contains more or less higher order contributions. In this sense there is no inaccuracy, but it is a different approximation.

\section{K. Ellis, Fermilab:}

You showed calculations due to Jung and Salam which lead to a larger beauty quark cross section at the TEVATRON. It is important to establish what feature of the calculations give rise to the increase. Does the unintegrated gluon distribution used in the calculations give (after integration over $k_{T}^{2}$ ) a gluon distribution in accord with the gluon distribution measured elsewhere at HERA ? Answer:

The gluon density is not a physical observable. Therefore the gluon as extracted from NLO DGLAP fits and from the CCFM scheme do not have to agree and in fact ther do not. The unintegrated gluon is higher 51 . However, it is only important that both describe the cross section measurements and in fact they both do so.

\section{References}

1. E.A. Kuraev, L.N. Lipatov, V.S. Fadin, Sov. Phys. JETP 44 (1976) 443 and Sov. Phys. JETP 45 (1977) 199; I.I. Balitsky, L.N. Lipatov, Sov. J. Nucl. Phys. 28 (1978) 822.

2. V.S. Fadin, L.N. Lipatov, Phys. Lett. B 429 (1998) 127 ; see also: G. Camici, M. Ciafaloni, Phys. Lett B 412 (1997) 396 (erratum ibid. B 417 (1997) 390 ; G. Camici, M. Ciafaloni, Phys. Lett. B 430 (1998) 349.

3. S.J. Brodsky et al., JETP Lett. 70 (1999); J. Blümlein, A. Vogt, Phys. Rev. D 57 
(1998) 1; ibid. D 58 (1998) 014020; C. Schmidt, Phys. Rev. D 60 (1999) 074003; J.R. Forshaw, D.A. Ross, A. Sabio Vera, Phys. Lett. B 455 (1999) 273; J. Kwiecinski, A.D. Martin, P. Sutton, Z. Phys. C 71 (1996) 585.

4. J. Kwiecinski, A.D. Martin, A. Stasto, Phys. Rev. D 56 (1997) 3991.

5. G. Salam, in: Cracow School of Theo. Phys. (1999) hep-ph/9910492; C.R. Schmidt, in: 5th Int. Sym. on Rad. Corr., Carmel (USA), hep-ph/0106181.

6. M. Ciafaloni, D. Colferai, Phys. Lett. B 452 (1999) 372; M. Ciafaloni, D. Colferai, G. Salam, Phys. Rev. D 60 (1999) 1140036; G. Salam, JHEP 07 (1998) 19.

7. V.N. Gribov, L.N. Lipatov, Sov. J. Nucl. Phys. 15 (1972) 438; G. Altarelli, G. Parisi, Nucl. Phys. B 126 (1977) 298; Yu. L. Dokshitzer, Sov. Phys. JETP 46 (1977) 641.

8. A. Martin, Lect. XXI Int. Meet. Fund. Phys., Madrid (1993) DTP/93/66.

9. A. De Rujula et al., Phys. Rev. D 10 (1974) 1649.

10. S. Catani, M. Ciafaloni, F. Hautmann, Nucl. Phys. B 366 (1991) 135; J.C. Collins, R.K. Ellis, Nucl. Phys. B 360 (1991) 3; E.M. Levin et al., Sov. J. Nucl. Phys. 54 (1991) 867.

11. M. Ciafaloni, Nucl. Phys. B 296 (1988) 49; S. Catani, F. Fiorani, G. Marchesini, Phys. Lett. B 234 (1990) 339 and Nucl. Phys. B 336 (1990) 18; G. Marchesini, Nucl. Phys. B 445 (1995) 49.

12. J. Bartels, S. Gieseke, C.F. Qiao, Phys. Rev. D 63 (2001) 056014 and hepph/0107152; V.S. Fadin, D. Yu. Ivanov, M. I. Kotsky, hep-ph/0106099.

13. M.A. Kimber, J. Kwiecinski, A.D. Martin, Phys. Lett. B 508 (2001) 58.

14. G. Marchesini, B. Webber, Nucl. Phys. B 349 (1991) 617 and Nucl. Phys. B 386 (1992) 215.

15. G. Bottazzi, G. Marchesini, G. Salam, M. Scorletti, Nucl. Phys. B 505 (1997) 366 and JHEP 9812 (1998) 011.

16. H. Jung, hep-ph/0109102, to be publ. Comp. Phys. Com.

17. H. Jung, G. Salam, Eur. Phys. J. C 19 (2001) 351.

18. B.Andersson et al., Nucl. Phys. B 467 (1996) 443; Z. Phys. C 71 (1996) 613; Nucl. Phys. B 467 (1996) 443; H. Kharrazhia, L. Lönnblad, JHEP 03 (1998) 6.
19. A.H. Mueller, H. Navelet, Nucl. Phys. B 282 (1987) 727.

20. D0 coll., Phys. Rev. Lett. 84 (2000) 5722.

21. L.H. Orr, W. J. Stirling, Phys. Lett. B 429 (1998) 127.

22. J.R. Andersen et al., JHEP 02 (2001) 007.

23. S. Frixione, G. Ridolfi, Nucl. Phys. B 507 (1997) 315; Kramer, Klasen, Phys. Lett. B 366 (1996) 385; H1 coll., Eur. Phys. J. C 13 (2000) 415; T. Carli, hep-ph/9906541.

24. J. Bartels, A. de Roeck, H. Lotter, Phys. Lett. B 389 (1996) 742; J. Bartels, C. Ewerz, R. Staritzbichler, Phys. Lett. B 492 (2000) 56.

25. S.J. Brodsky et al., JETP Lett. 70 (1999) 155; and V. Kim private comm.

26. L3 coll., Phys. Lett. B 453 (1999) 333.

27. L3 coll., L3 note 2680, LP01.

28. M. Cacciari et al., JHEP 0102 (2001) 029.

29. OPAL coll., hep-ex/0110006 subm. to Eur. Phys. J. C.

30. J. Kwiecinski, L. Motyka, Phys. Lett. B 462 (1999) 203.

31. H1 coll., Nucl. Phys. B 407 (1993) 515 and B 439 (1995) 471; Nucl. Phys. B 470 (1996) 3; Eur. Phys. J. C 13 (2000) 609. ZEUS coll., Phys. Lett. B 316 (1993) 412; Z. Phys. C 65 (1995) 379; and C 69 (1996) 607;

32. H1 coll., Eur. Phys. J. C 19 (2001) 269; and C 21 (2001) 33; ZEUS coll., Eur. Phys. J. C 21 (2001) 443.

33. M. Botje, Eur. Phys. J. C 10 (2000) 285; V. Barone, C. Pascaud, F. Zomer, hepph/0004268; ZEUS coll., contr. P1-628 to EPS01.

34. A.D. Martin et al., Eur. Phys. J. C 4 (1998) 463 and C 14 (2000) 13.

35. CTEQ coll., Eur. Phys. J. C 12 (2000) 375.

36. H1 coll., Phys. Lett. B 520 (2001) 183.

37. R. Thorne, Phys. Lett. B 474 (2000) 372 and Phys. Rev. D 64 (2001) 074005.

38. G. Altarelli, R.D. Ball, S. Forte, Nucl. Phys. B 599 (2001) 383.

39. A.D. Martin et al., hep-ph/0110215.

40. D0 coll., Phys. Rev. Lett. 86 (2001) 1707.

41. CDF coll., Phys. Rev. D 64 (2001) 032001.

42. R. Thorne, talk given at the Weimar QCD network meeting, 2001.

43. H1 coll., hep-ex/0108039.

44. B.W. Harris, J. Smith, Nucl. Phys. B 452 (1995) 109, Phys. Lett. B 353 (1995) 535, Phys. Rev. D 57 (1998) 2806.

45. C. Peterson et al., Phys. Rev. D 27 (1983) 105 . 
46. ZEUS coll., Eur. Phys. J. C 12 (2000) 35.

47. E. Norrbin, T. Sjöstrand, Eur. Phys. J. C 17 (2000) 137.

48. H1 collab, contr: 807, LP01

49. H1 coll., Phys. Lett. B 467 (1999) 156; erratum ibid B 518 (2001) 331.

50. ZEUS coll., Eur. Phys. J. C 18 (2001) 625.

51. H. Jung, hep-ph/0109146.

52. L3 coll., Phys. Lett. B 503 (2001) 10; OPAL note PN455.

53. CDF coll., Phys. Rev. D 55 (1997) 2546; D0 coll., Phys. Lett. B 487 (2000) 264; and Phys. Rev. Lett. 85 (2000) 5068.

54. M. L. Mangano, P. Nason, G. Ridolfi, Nucl. Phys. B 373 (1992) 295.

55. S. Frixione, M.L. Mangano, Nucl. Phys. B 483 (1997) 321.

56. H. Jung, hep-ph/0110034

57. P. Hägler et al., Phys. Rev. D 62 (2000) 071502

58. A.H. Mueller, Nucl. Phys. B (Proc. Suppl.) 18 C (1990) 125; J. Phys. G 17 (1991) 1443; J. Bartels, A. de Roeck, M. Loewe, Z. Phys. C 54 (1992) 635.

59. ZEUS collab, Phys. Lett. B 474 (2000) 1 and Eur. Phys. J. C 6 (1999) 239; H1 coll., Nucl. Phys. B 538 (1999) 3.

60. J. Bartels et al., Phys. Lett. B 384 (1996) 300.

61. J. Kwiecinski, A. Martin, J. Outhwaite, Eur. Phys. J. C 9 (1999) 611.

62. H. Jung, L. Jönsson, H. Küster, Eur. Phys. J C 9 (1999) 383.

63. G. Kramer, B. Pötter, Phys. Lett. B 453 (1999) 295.

64. T. Schoerner, DIS 2000.

65. M. Kuhlen, Phys. Lett. B 382 (1996) 441.

66. H1 coll., Phys. Lett. B 462 (1999) 440.

67. ZEUS coll., Phys. Lett. B 315 (1993) 481 and B 332 (1994) 228; Z. Phys. C 68 (1995) 569; Eur. Phys. J. C 1 (1998) 81 and C 6 (1999) 43; H1 coll., Nucl. Phys. B 429 (1994) 477; Phys. Lett. B 348 (1995) 681; Nucl. Phys. B 472 (1996) 3; Z. Phys. C 69 (1995) 27 and C 75 (1997) 607 and C 76 (1997) 613.

68. K. Goulianos, hep-ex/0011060, J. Bartels and H. Kowalski, hep-ph/0010345.

69. H1 coll., contr: 200, LP01

70. ZEUS coll., Phys. Lett. B 315 (1993) 481.

71. M. Wüsthoff, A.D. Martin, J. Phys. G 25 (1999) R309, M.F. McDermott, hepph/0008260; A. Hebecker, Acta Phys. Polon. B 30 (199) 3777 and Phys. Rep.
331 (2000) 1.

72. F.E. Low, Phys. Rev. D 12 (1975) 163; S. Nussinov, Phys. Rev. 34 (1976) 1286; J. Gunion, D.E. Soper, Phys. Rev. D 15 (1977) 2617; E.M. Levin, M.G. Ryskin, Sov. J. Nucl. Phys. 34 (1981) 619.

73. A. Donnachie, P.V. Landshoff, Phys. Lett. B 296 (1992) 227.

74. ZEUS coll., Phys. Lett. B 487 (2000) 1 and EPS01.

75. ZEUS coll., Phys. Rev. D 53 (2000) 53.

76. H1 coll., Z. Phys. C 69 (1995) 27; ZEUS coll., Z. Phys. C 63 (1994) 391 and contr: 1046, ICHEP 2000.

77. N. Nikolaev, B.G. Zakharov, Z. Phys. C 49 (1990) 607 and Z. Phys. C 53 (1992) 331; A.H. Mueller, Nucl. Phys. B 415 (1994) 373 and Nucl. Phys. B 437 (1995) 107;

78. A. Bialas, H. Navelet, R. Peschanski, Nucl. Phys. B 593 (2001) 438.

79. K. Golec-Biernat, M. Wüsthoff, Phys. Rev. D 59 (1999) 014017.

80. K. Golec-Biernat, M. Wüsthoff, Phys. Rev. D 60 (1999) 114023.

81. K. Golec-Biernat, hep-ph/0109010.

82. G. Iaccobucci, these proceedings.

83. A. Belavin et al., Phys. Lett. B 59 (1975) 85; G. t'Hooft, Phys. Rev. D 14 (1976) 3432 and Phys. Rev. Lett. 37 (1976) 8.

84. S. Moch, A. Ringwald, F. Schrempp, Nucl. Phys. B 507 (1997) 134.

85. A. Ringwald,F. Schrempp, hep-ph/9411217.

86. A. Ringwald, F. Schrempp, Phys. Lett. B 459 (1999) 249.

87. A. Ringwald, F. Schrempp, Phys. Lett. B 438 (1998) 217.

88. A. Ringwald,F. Schrempp, hep-ph/9909338, hep-ph/0006215

89. A. Ringwald, F. Schrempp, Comp. Phys. Commun. 132 (2000) 267.

90. T. Carli et al., hep-ph/9906441

91. H1 coll., contr. paper to ICHEP00.

92. T. Carli, M. Kuhlen, Nucl. Phys. B 511 (1998) 85.

93. A. Ringwald, F. Schrempp, Phys. Lett. B 503 (2001) 331. 